\title{
Review of: "Genome of the pincer wasp Gonatopus flavifemur reveals unique venom evolution and a dual adaptation to parasitism and predation"
}

\author{
zhiqiang $\mathrm{Lu}^{1}$ \\ 1 Northwest A \& F University
}

Potential competing interests: The author(s) declared that no potential competing interests exist.

Strength: comprehensive bioinformatics data analysis and provided significant insights.

Weakness: Lack of bench work data to support these suggestions. 